\title{
MRI in the Follow-up of Testicular Cancer: Less is More
}

\author{
ERIK RUD ${ }^{1}$, CARL WILHELM LANGBERG $^{2}$, EDUARD BACO $^{3}$, PETER LAURITZEN $^{1}$ and GUNNAR SANDB EK $^{1}$ \\ ${ }^{1}$ Department of Radiology and Nuclear Medicine, Oslo University Hospital, Oslo, Norway; \\ ${ }^{2}$ Department of Oncology, Oslo University Hospital, Oslo, Norway; \\ ${ }^{3}$ Department of Urology, Oslo University Hospital, Oslo, Norway
}

\begin{abstract}
Background/Aim: This study aimed to report the location of abdominal relapse in patients with testicular cancer. Materials and Methods: This is a retrospective cross-sectional study including patients who underwent abdominal magnetic resonance imaging (MRI) after treatment of testicular germ cell cancer. MRI reports were classified as negative or positive, and positive results were cross-checked with follow-up imaging and biopsy results. Positive histology or cytology defined a true-positive finding. The location of relapse was registered according to the anatomical site. Results: In a 2-year period, 2,315 MRI examinations were performed. Relapse was detected in $0.7 \%$ (95\% CI=0.4-1.1) of the examinations. Among these, $75 \%$ were seminomas and $25 \%$ were non-seminomas. Retroperitoneal lymph nodes were affected in $88 \%$ of cases, and pelvic and inguinal lymph nodes affected in $12 \%$ of cases. No metastases were found in parenchymatous organs or bony structures. Conclusion: All cases of abdominal relapse occurred in retroperitoneal or pelvic lymph nodes. This suggests that MRI should be directed towards the retroperitoneum and pelvis only.
\end{abstract}

Patients are monitored for relapse for 5 to 10 years after orchidectomy, although relapse is rare after two years for nonseminomas and three years for seminomas (1). The European Society of Medical Oncology (ESMO) recommends magnetic resonance imaging (MRI) of the retroperitoneum in order to reduce the radiation dose (2). However, the level of evidence is weak (III), and no studies have specifically reported the primary site of relapse in these patients.

Retroperitoneal lymph nodes are the most common site of relapse (3). Metastases to the liver, bones or lungs have also

Correspondence to: Erik Rud, MD, Ph.D., Department of Radiology and Nuclear Medicine, Oslo University Hospital, Trondheimsveien 235, 0514 Oslo, Norway. Tel: +47 23034534, Fax: +47 23033046, e-mail: erik.rud@ous-hf.no

Key Words: MRI, testicular cancer, seminoma, non-seminoma, relapse, lymph node, metastases. been reported, but the presence of concomitant nodal metastases in these cases has not been specified (4-6). Our current MRI examination is based on the recommendation of the Swedish-Norwegian Testicular Cancer group. It is designed to evaluate lymph nodes, all abdominal parenchymatous organs, as well as the bony structures from the diaphragm to the proximal femur.

The aim of the study was to identify the location of testicular cancer relapses in the abdomen, and to assess whether a targeted MRI evaluation of pelvic and retroperitoneal lymph nodes is sufficient to monitor these patients.

\section{Materials and Methods}

This is a retrospective cross-sectional diagnostic study approved by the Patient Protection Council (18/13813). A waiver was issued for informed consent. Using the radiological information system, all patients that underwent abdominal MRI after being diagnosed with testicular cancer during a two-year period were identified. All disease stages according to Royal Marsden were included, and all were monitored according to the follow-up program defined by Swedish Norwegian Testicular Cancer group (SWENOTECA). MRI examinations were performed at 1.5T Intera Phillips with sequences according to Table I.

The results of the original report were used. Metastases to the lymph nodes were suspected in case of short axis $>8 \mathrm{~mm}$, and/or long axis $>10 \mathrm{~mm}$ (7). Measurements were made in the transversal plane. There were no predefined criteria for metastases to abdominal organs or bony structures. This assessment was at the discretion of the reading radiologist.

Patients were dichotomized into those with and those without a relapse. A true positive finding was defined as positive MRI and positive biopsy or cytology. When biopsy or cytology was not possible for technical reasons, a positive 18-fluorodeoxyglucose positron emission tomography (FDG-PET) CT followed by chemotherapy and/or radiation therapy was defined as a true positive. In cases of inconclusive MRI reports, follow up images and/or biopsies were used as reference standard. Patients with relapse were included for further analyses.

Time to relapse was defined as the number of months from orchidectomy to the date of positive MRI. In patients with relapse, age, clinical disease stage at the time of diagnosis, histological type (seminoma or non-seminoma), and presence of risk factors for relapse (testicular tumor size, invasion of the rete testis and vascular invasion) were registered. 
We registered if relapsing patients had received adjuvant therapy or not, and checked if there was any difference in time to relapse between the two groups. Furthermore, differences in tumor size, age, or time to relapse between seminomas and non-seminomas were examined.

Statistical methods. Mann-Whitney $U$-test was used to assess the significance of differences. For non-normally distributed data, median values with interquartile range (IQR) are reported. The significance level was defined as $p<0.05$. SPSS v.25 (IBM $\left.{ }^{\circledR}\right)$ was used for statistical analyses.

\section{Results}

MRI detected relapse in 16 patients out of 2315 MRI examinations $(0.7 \%, 95 \% \mathrm{CI}=0.4-1.1)$. Among these, $75 \%$ were seminomas (12 patients); ten were stage 1 and two were stage 2 . The remaining $25 \%$ (4 patients) were stage 1 non-seminomas. Five patients with seminomas and one patient with non-seminoma had received adjuvant therapy.

Relapse in retroperitoneal lymph nodes occurred in $88 \%$ (14 patietns) of cases, and in pelvic or inguinal lymph nodes in $12 \%$ (2) of cases (Figures 1-4). No relapses were found in other abdominal organs or bony structures. The median short and long axis of the metastatic lymph nodes were $17 \mathrm{~mm}$ $(\mathrm{IQR}=15-21$, range $=8-33 \mathrm{~mm})$ and $20 \mathrm{~mm}(\mathrm{IQR}=18-25$, range $=10-55 \mathrm{~mm}$ ), respectively.

Relapse was verified by a positive biopsy or cytology in $81 \%$ (13) of cases, and by a positive FDG-PET CT in $19 \%$ (3) of cases for which the lymph nodes were inaccessible for biopsy or cytology. A total of 143 MRI examinations were needed in order to find one case of relapse.

In patients with relapse, the median testicular tumor size was $38 \mathrm{~mm}$ (IQR=22-58, range 17-73, unknown in two), and there was no difference between seminomas and nonseminomas $(p=0.3)$. In case of seminomas, $50 \%(6)$ of the relapsing patients demonstrated tumor size $>40 \mathrm{~mm}$, and $30 \%$ (4) showed invasion of the rete testis. Four patients exhibited both risk factors. In case of non-seminomas, none of the relapsing patients showed vascular invasion.

The median age at the time of relapse was 41 years (IQR=30-45, range=16-73 years), and there was no difference between seminomas and non-seminomas $(p=0.3)$. The overall median time to relapse was 11 months $(\mathrm{IQR}=7-20$, range=496 months). In seminomas and non-seminomas, the time to relapse was 13 months (IQR=9-30) and 7 months (IQR=5-9), respectively ( $p=0.020)$. In two patients, relapse occurred at 3 years or later, after orchidectomy. There was no difference in the time to relapse between patients who received adjuvant therapy compared to those who did not $(p=0.3)$.

\section{Discussion}

This study demonstrated that all cases of abdominal relapse occurred in lymph nodes. This suggests that the MRI
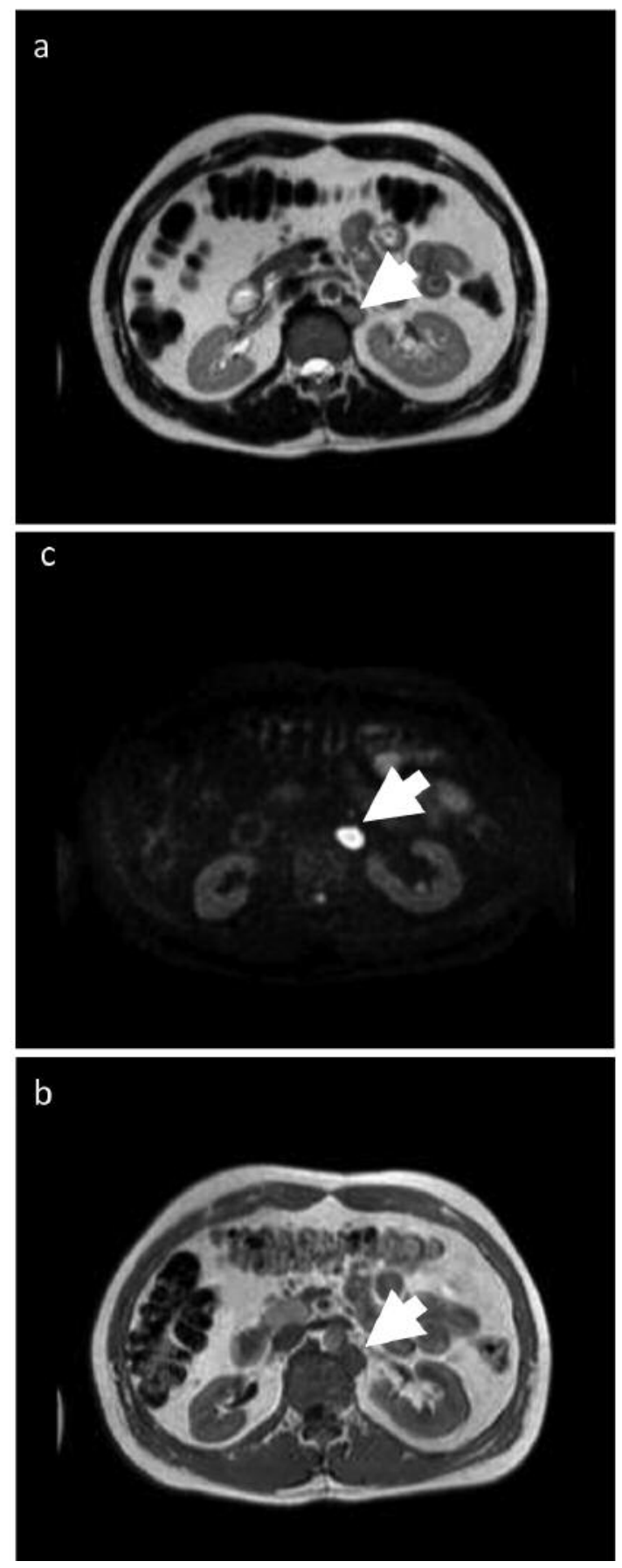

Figure 1. A 32-year-old patient with a 25-mm seminoma on the left side without invasion of the rete testis, clinical stage 1. Relapse developed 13 months after surgery on the retroperitoneal wall (red arrow) at the level of the left renal vein. T2w (a), T1w (b), and diffusion weighted images (c) demonstrate a 2-cm metastatic lymph node confirmed upon histology.

protocol should focus at the retroperitoneal lymph nodes only rather than at abdominal organs and bony structures. Such a simplification would reduce the MRI scanning from 

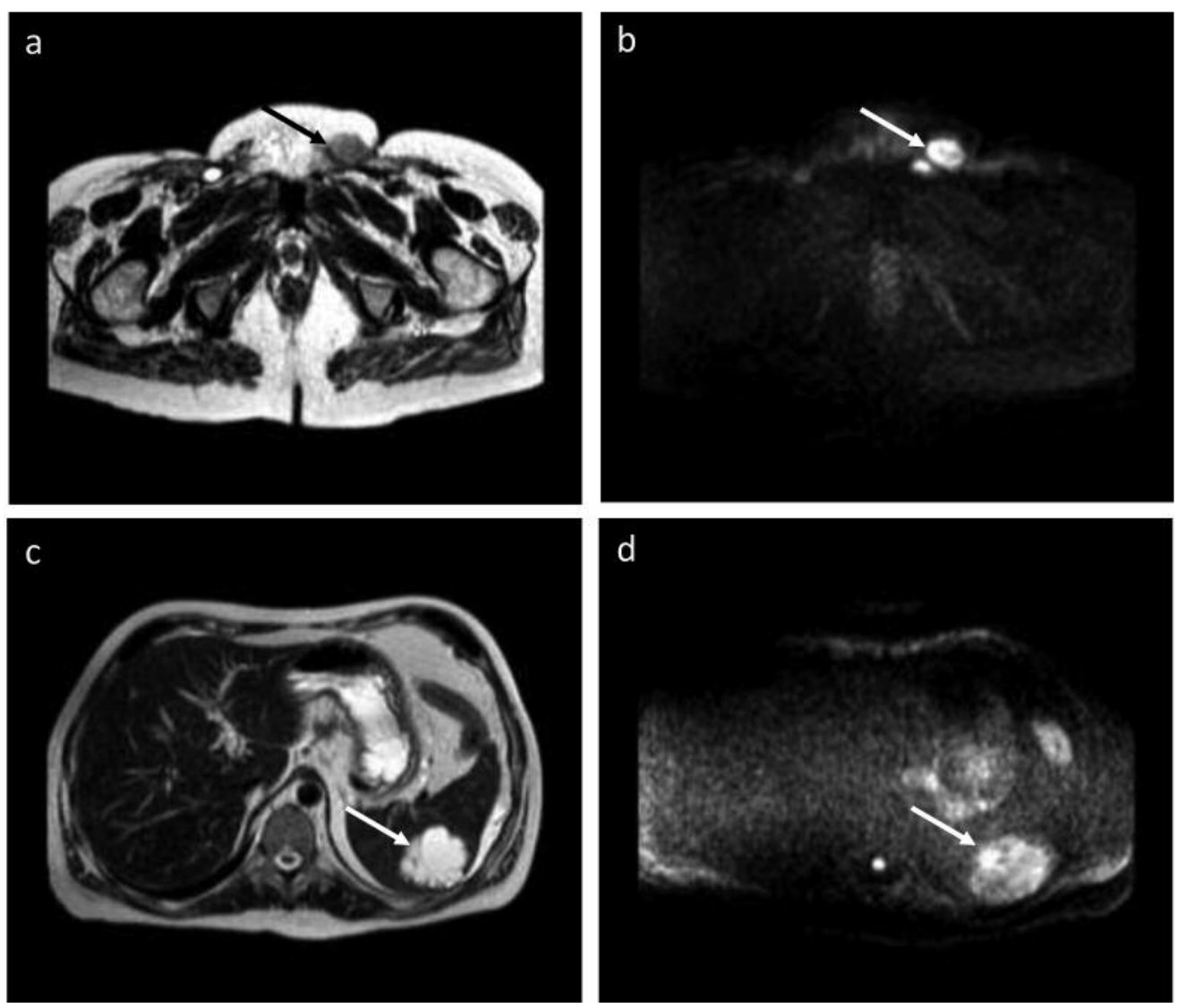

Figure 2. A 44-year-old patient with clinical stage $2 b$ seminoma on the left side. Tumor size and rete testis invasion status were unknown since the patient was initially treated abroad. Relapse developed in the left iliac region/testicular funicle after 12 months. This is an uncommon site of relapse. It is unknown if the patient previously had undergone inguinal hernia surgery which is known to alter the lymphatic drainage, and possibly favor inguinal lymph node metastases. A T2w image (a) and diffusion-weighted image using $b 800(b)$ demonstrate a 2-cm lymph node in the left pelvic region. Histology confirmed relapse. In addition, T2w image $(c)$ and b800 (d) demonstrated a cystic mass in the spleen that turned out to be benign after splenectomy.

Table I. MRI sequences used for monitoring relapse.

\begin{tabular}{lccc}
\hline Weighting & Plane of acquisition & Respiration & Details \\
\hline T2 & Coronal & Free breathing & Coronal Half acquisition single hot turbo-spin echo sequence \\
T2 & Axial & Triggered & Axial T2 weighted turbo-spin echo sequence \\
T1 & Axial & Breath hold & Gradient-echo sequence with turbo-spin echo sequence \\
DWI & Axial & Free breathing & b0, b50, b800 with apparent diffusion coefficient map \\
STIR & Coronal & Free breathing & \\
\hline
\end{tabular}

The scanned volume includes the abdominal cavity until the proximal femur. Scanning time is approximately 30 min but depends on the MRI manufacturer. The respiratory rate of the patients significantly influences the scanning time. The different sequences have different names and acquisition details depending on the manufacturer.

30-35 $\min$ to $12-13 \mathrm{~min}$ as only one anatomical sequence and diffusion weighted images (DWI) would probably be sufficient to detect lymph nodes. A short tau inversion recovery (STIR) sequence for detecting bone metastases, is on the other hand redundant, as bone metastases were not seen in any patients. The importance of the retroperitoneal lymph nodes is also emphasized by European Society of Medical Oncology (ESMO) (2). 

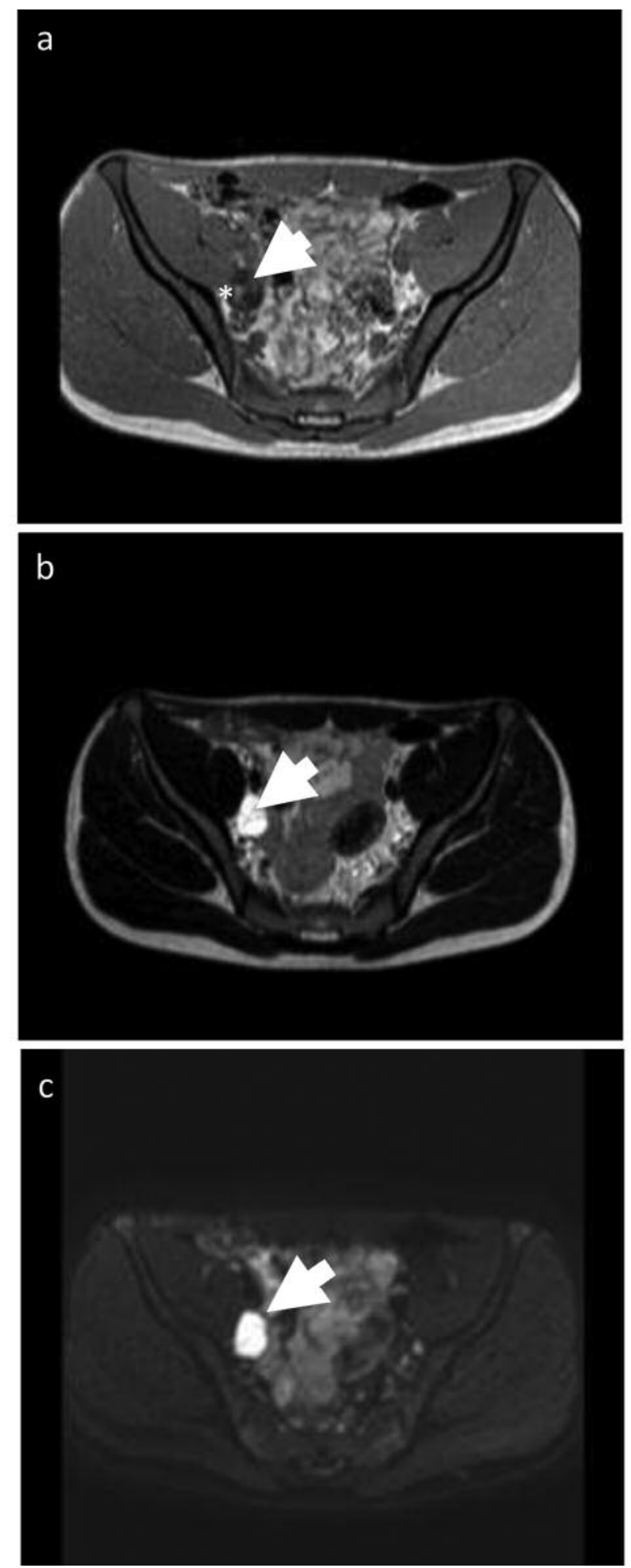

Figure 3. A 36-year-old patient with stage 1, non-seminoma without vascular invasion. A 2.5-cm relapse developed on the pelvic wall 8 months after surgery as seen on $T 1 w(a), T 2 w(b)$ and diffusion weighted images [b800] (c). The lesion was mainly cystic as seen on the bright signal on $T 2 w$ image. The tumor also contained fat, indicated with the yellow star on the T1w image (a). Histology confirmed a malignant teratoma.
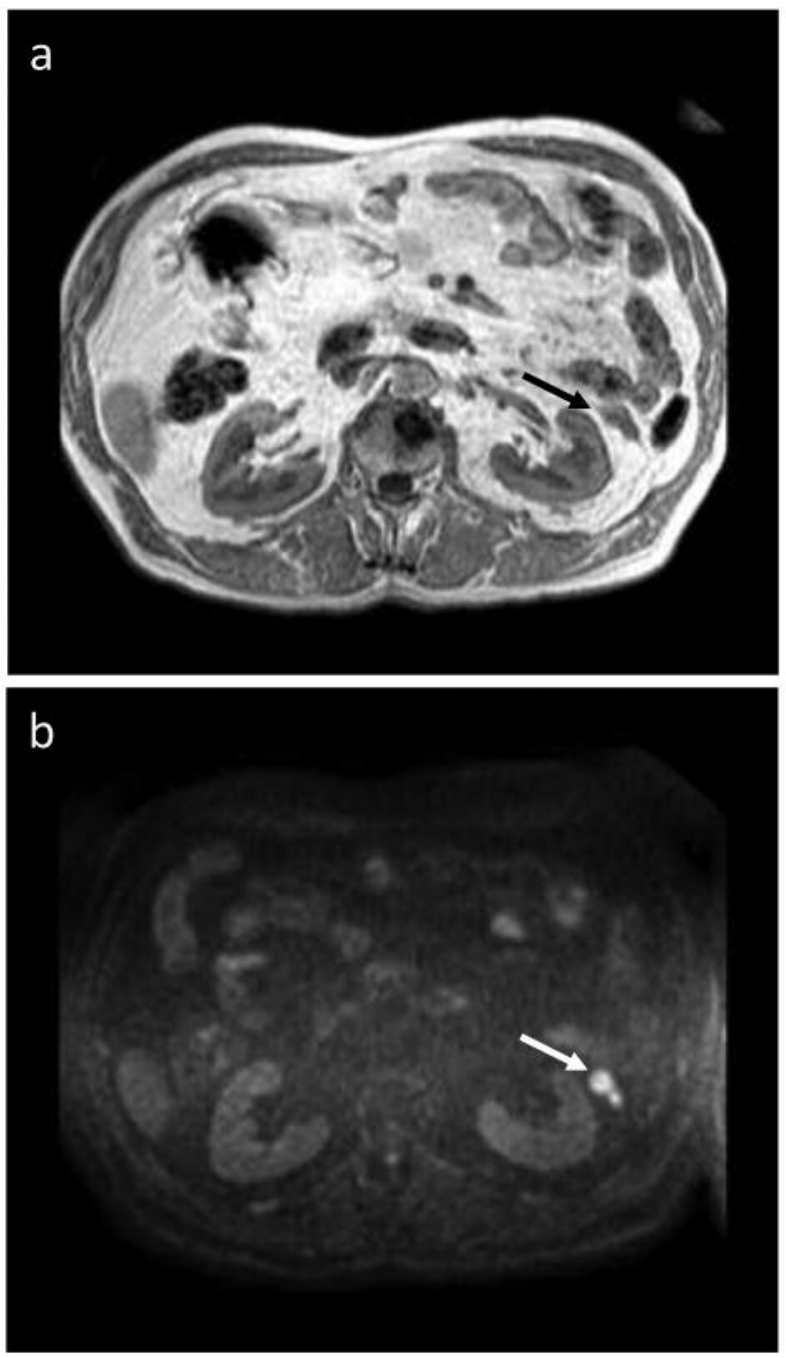

Figure 4. A 73-year-old patient with a 65-mm seminoma with an invasion of the rete testis. Preoperative CT detected a 17-mm retroperitoneal lymph node metastasis (clinical stage 2a). Orchidectomy was performed with adjuvant external beam radiation (27 Gy). After 18 months, MRI detected two enlarged lymph nodes at the retroperitoneal wall along the Gerota's fascia. Relapse lateral to the typical aorto-caval lymph nodes are unusual, and it is believed to occur more frequently in cases of relapse rather than at the initial staging. T1w (a) and diffusionweighted image $(b)$.

Although relapses are reported in organs other than lymph nodes, these studies do not specify whether extranodal metastases occurred alone or with concomitant nodal metastases $(4,8,9)$. However, it is important to know whether the extra nodal metastases were present at the time of primary diagnosis, since these patients invariably have a more serious disease, and should in our opinion, be monitored more thoroughly with contrast enhanced CT. 
In our study, the median short axis of the metastatic lymph nodes was $16 \mathrm{~mm}$ (range $=8-33 \mathrm{~mm}$ ) and the long axis was $20 \mathrm{~mm}$ (range=10-55 $\mathrm{mm}$ ). The Swedish Norwegian Testicular Cancer group (SWENOTECA) defines enlarged lymph nodes as more than $10 \times 8 \mathrm{~mm}$, while other studies use only the short axis diameter $>10 \mathrm{~mm}(7,10)$. Using size criteria to define a metastatic lymph node is problematic for several reasons. First of all, the normal size is different in different body regions, and it is well known that a significant number of metastases occur in small- and normal-sized lymph nodes (11). Others have shown an incremental value of evaluating the presence of hilar fat, contrast-enhancement, and shape of lymph nodes on MRI (12), but in the end, there are no reliable criteria for distinguishing benign from malignant lymph nodes. Therefore, the true prevalence of lymph node metastases is difficult to verify in a crosssectional study such as ours. Since size was the only criteria for defining metastasis, one may also argue that DWI is excessive. However, some suggest that DWI makes it easier to detect lymph nodes and Mosavi et al., recently demonstrated the potential important role of DWI when investigating testicular cancer relapse (13). Our study cannot assess the isolated value of DWI for detecting lymph node metastases.

What is preferable; CT or MRI?. The most obvious benefit of MRI over CT is the absence of ionizing radiation and absence of intravenous contrast medium. Patients are usually young, and they will undergo multiple radiological examinations during follow-up.

Previous studies have reported that MRI is equal to CT in the primary workup for detecting retroperitoneal lymph node metastases $(14,15)$. In 2018, ESMO recommended contrast enhanced CT in the initial staging and MRI of the retroperitoneum in the follow-up after treatment (2).

Limitations. There are some limitations to this study. First of all, we cannot assess the rate of metastases to the lungs and the rate of biochemical relapse. The current study is a crosssectional study including patients referred for abdominal MRI only. Consequently, the overall relapse rate over time cannot be assessed.

Since there were few cases of relapse in this study, unexpected locations of relapse could occur if more patients were included. However, the probability of extra-nodal metastases remains extremely low $(<1 / 2,351)$. Last, due to the retrospective study design the clinical follow-up of unspecific findings was not standardized.

In conclusion, the site of abdominal relapse in patients with testicular cancer is highly predictable, as all cases occurred in retroperitoneal or pelvic lymph nodes. This evidence suggests that MRI examination should focus on the retroperitoneum only.

\section{Conflicts of Interest}

The Authors have no conflicts of interest to declare regarding this study.

\section{Authors' Contributions}

ER: Project development, data collection, manuscript writing and editing; CWL: Data collection, manuscript writing and editing; EB: Manuscript writing and editing; PML: Manuscript writing and editing; GS: Manuscript writing and editing.

\section{Acknowledgements}

The Authors would like to thank the radiographer Gøril Meland for identifying all patients who underwent MRI during the study period.

\section{References}

1 Kollmannsberger C, Tandstad T, Bedard PL, Cohn-Cedermark G, Chung PW, Jewett MA, Powles T, Warde PR, Daneshmand S, Protheroe A, Tyldesley S, Black PC, Chi K, So AI, Moore MJ and Nichols CR: Patterns of relapse in patients with clinical stage I testicular cancer managed with active surveillance. J Clin Oncol 33: 51-57, 2015. PMID: 25135991. DOI: 10.1200/ JCO.2014.56.2116

2 Honecker F, Aparicio J, Berney D, Beyer J, Bokemeyer C, Cathomas R, Clarke N, Cohn-Cedermark G, Daugaard G, Dieckmann KP, Fizazi K, Fosså S, Germà-Lluch JR, Giannatempo P, Gietema JA, Gillessen S, Haugnes HS, Heidenreich A, Hemminki K, Huddart R, Jewett MAS, Joly F, Lauritsen J, Lorch A, Necchi A, Nicolai N, Oing C, Oldenburg J, Ondruš D, Papachristofilou A, Powles T, Sohaib A, Stahl O, Tandstad $\mathrm{T}$, Toner $\mathrm{G}$ and Horwich A: ESMO Consensus Conference on testicular germ cell cancer: diagnosis, treatment and follow-up. Ann Oncol 29: 1658-1686, 2018. PMID: 30113631. DOI: 10.1093/annonc/mdy217

3 Barrisford GW, Kreydin EI, Preston MA, Rodriguez D, Harisighani MG and Feldman AS: Role of imaging in testicular cancer: Current and future practice. Future Oncol 11: 2575-2586, 2015. PMID: 26314944. DOI: 10.2217/fon.15.194

4 Oldenburg J, Martin JM and Fosså SD: Late relapses of germ cell malignancies: incidence, management, and prognosis. J Clin Oncol 24: 5503-5511, 2006. PMID: 17158535. DOI: 10.1200/ JCO.2006.08.1836

5 La Pena De H, Sharma A, Glicksman C, Joseph J, Subesinghe M, Traill Z, Verrill C, Sullivan M, Redgwell J, Bataillard E, Pintus E, Dallas N, Gogbashian A, Tuthill M, Protheroe A and Hall M: No longer any role for routine follow-up chest $\mathrm{x}$-rays in men with stage I germ cell cancer. Eur J Cancer 84: 354-359, 2017. PMID: 2886637. DOI: 10.1016/j.ejca.2017.07.005

6 Dieckmann K, Albers P, Classen J, Dewit M, Pichlmeie U, Rick O, Mullerleile U and Kuczyk M. Late relapse of testicular germ cell neoplasms: A descriptive analysis of 122 cases. J Urol 173: 824-829, 2005. PMID: 15711278. DOI: 10.1097/01.ju.0000 154013.96349 .36

7 Forsberg L, Dale L, Høiem L, Magnusson A, Mikulowski P, Olsson AM, Ous S and Stenwig AE: Computed tomography in early stages of testicular carcinoma. Size of normal retroperitoneal 
lymph nodes and lymph nodes in patients with metastases in stage II A. A SWENOTECA study: Swedish-Norwegian Testicular Cancer Project. Acta Radiol Diagn (Stockh) 27: 569-574, 1986. PMID: 3026141.

8 Albers P, Siener R, Kliesch S, Weissbach L, Krege S, Sparwasser C, Schulze H, Heidenreich A, de Riese W, Loy V, Bierhoff E, Wittekind C, Fimmers R, Hartmann MGerman Testicular Cancer Study Group: Risk factors for relapse in clinical stage I nonseminomatous testicular germ cell tumors: results of the German Testicular Cancer Study Group Trial. J Clin Oncol 21: 1505-1512, 2003. PMID: 12697874. DOI: 10.1200/JCO.2003.07.169

9 Cohn-Cedermark G, Stahl O, Tandstad T SWENOTECA: Surveillance vs. adjuvant therapy of clinical stage I testicular tumors - a review and the SWENOTECA experience. Andrology 3: 102-110, 2014. PMID: 25270123. DOI: 10.1111/andr.280

10 Heidenreich A, Albers P, Classen J, Graefen M, Gschwend J, Kotzerke J, Krege S, Lehmann J, Rohde D, Schmidberger H, Uder M and Zeeb H; Association of Urologic Oncology of the German Cancer Society: Imaging studies in metastatic urogenital cancer patients undergoing systemic therapy: recommendations of a multidisciplinary consensus meeting of the Association of Urological Oncology of the German Cancer Society. Urol Int 85: 1-10, 2010. PMID: 20693823. DOI: 10.1159/000318985

11 Hilton S, Herr HW, Teitcher JB, Begg CB and Castéllino RA: $\mathrm{CT}$ detection of retroperitoneal lymph node metastases in patients with clinical stage I testicular nonseminomatous germ cell cancer: assessment of size and distribution criteria. AJR Am J Roentgenol 169: 521-525, 1997. PMID: 9242768. DOI: 10.2214/ajr.169.2.9242768
12 de Bondt RBJ, Nelemans PJ, Bakers F, Casselman JW, PeutzKootstra C, Kremer B, Hofman PAM and Beets-Tan RGH: Morphological MRI criteria improve the detection of lymph node metastases in head and neck squamous cell carcinoma: multivariate logistic regression analysis of MRI features of cervical lymph nodes. Eur Radiol 19: 626-633, 2009. PMID: 18839178. DOI: $10.1007 / \mathrm{s} 00330-008-1187-3$

13. Mosavi F, Laurell A and Ahlström H: Whole body MRI, including diffusion-weighted imaging in follow-up of patients with testicular cancer. Acta Oncol 54(10): 1763-1769, 2015. PMID: 25975210. DOI: 10.3109/0284186X.2015.1043027

14 Sohaib SA, Koh DM, Barbachano Y, Parikh J, Husband JES, Dearnaley DP, Horwich A and Huddart R: Prospective assessment of MRI for imaging retroperitoneal metastases from testicular germ cell tumours. Clin Radiol 64: 362-367, 2009. PMID: 19264179. DOI: 10.1016/j.crad.2008.10.011

15 Kok HK, Leong S and Torreggiani WC: Is magnetic resonance imaging comparable with computed tomography in the diagnosis of retroperitoneal metastasis in patients with testicular cancer? Can Assoc Radiol J 65: 196-198, 2014. PMID: 24054831. DOI: 10.1016/j.carj.2013.05.005
Received May 3, 2019

Revised May 16, 2019

Accepted May 17, 2019 